\title{
Application of a linear finite-frequency theory to time-lapse crosswell tomography in ultrasonic and numerical experiments
}

\author{
Jesper Spetzler ${ }^{1}$, Danijela Šijačić ${ }^{1}$, and Karl-Heinz Wolf ${ }^{1}$
}

\begin{abstract}
Time-lapse seismic monitoring is the geophysical discipline whereby multiple data sets recorded at the same location but at different times are used to locate and quantify temporal changes in the elastic parameters of the subsurface. We validate a time-lapse monitoring method by crosswell tomography using two types of wavefield-modeling experiments: (1) a 3D real ultrasonic waveform experiment and (2) 2D synthetic finite-difference wavefield simulations. For both wavefield experiments, a time-lapse structure simulating a fluid sweep in a reservoir layer is applied. The timelapse tomographic monitoring approach is based on the standard ray theory and a finite-frequency wavefield theory, where the latter takes into account the finite-frequency properties of recorded wavefields. The inverted time-lapse models compiled with either the ray theory or the finite-frequency wavefield theory locate and correctly quantify the flooding zone in the simulated fluid sweep model. Both wavefield theories provide an adequate result because the flooding zone is comparable in size to the Fresnel volume.
\end{abstract}

\section{INTRODUCTION}

Time-lapse (also known as 4D) seismic monitoring is not restricted only to reflection seismic data (see Sønneland et al., 1997; Landrø et al., 1999; Boyd-Gorst et al., 2001; Landrø, 2001; Guilbot and Smith, 2002; Bertrand and MacBeth, 2003). It is likewise possible to use transmission data recorded at the same location but at different times to assess the 4D changes of the subsurface elastic properties. Mostly, the tomography method is applied in transmission experiments to estimate the velocity structure of the target zone between the source and the receiver well. The seismic tomographic approach can be extended to a time-lapse mode in which the phase differences between the first and repeated transmission data are measured and used directly in a tomographic inversion. In turn, the inverted tomographic model indicates and quantifies the $4 \mathrm{D}$ velocity differences in the target zone between the source and the receiver well.

We apply a common tomographic scheme in our 4D monitoring method. To describe the physics of wave propagation in complex media, we use two approaches: (1) the standard ray theory (i.e., a high-frequency limit approach) wherein ray-bending effects are taken into account and (2) a linear finite-frequency wavefield theory that takes the finite-frequency characteristics of recorded seismic waves into account. The regime of the finite-frequency wavefield theory is extended to media with time-lapse anomaly structures smaller than the Fresnel volume (Woodward, 1992; Spetzler and Snieder, 2001, 2004). In contrast, the ray theory is only valid in media with 4D velocity changes larger than the Fresnel volume and the wavelength (Červený, 2001).

Tomography has been used in real time-lapse crosswell experiments. Paulsson et al. (1994) use crosswell data to monitor a tar (i.e., heavy oil) sand reservoir for the effect of steam injection. A tomographic ray-based method is used to estimate the velocity structure of the zone with steam injection at the time of the first and repeated crosswell surveys. The velocity of the tar sand formation decreases, which agrees with rock physics modeling (i.e., a temperature increase in a tar sand reservoir decreases the acoustic velocity). Saito et al. (2006) also apply ray-based tomography to monitor a sandstone reservoir for $\mathrm{CO}_{2}$ injection. They find a clear 4D velocity anomaly below the $\mathrm{CO}_{2}$ wellhead location. Velocity estimation by tomography is used on reflection data as well. For instance, the stereotomographic method is applied on $2 \mathrm{D}$ and $3 \mathrm{D}$ surface data (Billette et al., 2003), and Gao et al. (2003) use waveform tomography on combined VSP surface data. Vesnaver et al. (2003) show a case study of time-lapse reflection tomography applied in a North Sea experiment.

The time-lapse tomographic method is based on the ray theory and a linear finite-frequency wavefield theory. Hung et al. (2001) and Jocker et al. (2006) use synthetic forward modeling and real ultrasonic waveform experiments to validate the ray theory and the finite-frequency wavefield theory using a model with a spherical ve-

Manuscript received by the Editor 24 November 2006; revised manuscript received 8 June 2007; published online 26 September 2007.

${ }^{1}$ Delft University of Technology, Department of Geotechnology, Delft, the Netherlands. E-mail: j.spetzler@tudelft.nl; d.sijacic@tudelft.nl; k.h.a.a.wolf@ tudelft.nl.

(C) 2007 Society of Exploration Geophysicists. All rights reserved. 
locity anomaly surrounded by a homogeneous reference medium. The diameter of the sphere is smaller than the Fresnel volume; hence, the conditions for the ray theory are violated deliberately. Both papers clearly demonstrate by wavefield-modeling experiments that the ray theory overestimates the observed traveltime delays inherent in the velocity perturbation field (e.g., on an order of magnitude of two for 2D wave propagation and several orders higher for 3D wave propagation), whereas the finite-frequency wavefield theory reproduces the observations in a reasonable manner.

We apply a 2D synthetic-wavefield finite-difference modeling experiment and a 3D real ultrasonic waveform experiment to validate the inverse problem of the 4D tomographic method. The ultrasonic waveform recordings are from Legget et al. (1993), who built a timelapse set of two scaled epoxy models to simulate a fluid sweep in a reservoir layer. To generate a realistic flooding situation, half of the epoxy material in a layer of one of the blocks was replaced by another epoxy material with a lower acoustic velocity. Legget et al. use tomography based on the ray theory to estimate the pre- and postflood model. After estimated model differencing, their model shows a clear 4D anomaly located correctly according to the structure of the scaled epoxy blocks.

We extend the work of Legget et al. (1993) in several aspects: First, the measured time-lapse traveltime delays between the ultrasonic waveforms inherent to the flooding zone are used in a direct tomographic inversion to image the 4D velocity structure. Second, the time-lapse monitoring approach by tomography takes the finite-frequency properties of recorded wavefields into account because the linear finite-frequency wavefield theory is implemented in the forward problem. In addition, we supplement the 3D ultrasonic waveform experiment with $2 \mathrm{D}$ finite-difference wavefield simulations using the same (time-lapse) velocity structure as in the real epoxy models to expose the developed 4D tomographic algorithm to an extra test.

In the following sections, we describe our methodology for the 4D tomographic monitoring approach. Then, we present the 3D ultrasonic waveform data-validation experiment. Next, the verification test by $2 \mathrm{D}$ synthetic finite-difference wavefield simulations is explained. Last, we draw conclusions and discuss applications of the 4D monitoring method by tomography.

\section{METHODOLOGY FOR 4D MONITORING BY TOMOGRAPHY}

Time-lapse seismic monitoring differs from common seismic tomography in that two data sets are used instead of a single data set. The data set from a baseline survey is called the baseline data. At a later time, the baseline survey is repeated by a monitor survey, resulting in the monitor data set. Proper care must be taken to minimize the nonrepeatability effects from differences in source-receiver geometry and source wavelet signature. By applying the differences between the baseline and monitor data, we can estimate the location and quantify the temporal changes in the subsurface between the two survey times.

The scheme for time-lapse tomography is similar to the one for common traveltime delay tomography. There are four main steps in the time-lapse tomographic process. First, we estimate the timelapse time delay between the first-arrival times in the monitor and baseline data, $t_{\text {mon }}$ and $t_{\text {base }}$, respectively. The $4 \mathrm{D}$ time shift $\Delta t_{4 \mathrm{D}}$ for source position $\mathbf{r}_{s}$ and receiver position $\mathbf{r}_{r}$ is given by

$$
\Delta t_{4 \mathrm{D}}\left(\mathbf{r}_{r}, \mathbf{r}_{s}\right)=t_{\text {mon }}\left(\mathbf{r}_{r}, \mathbf{r}_{s}\right)-t_{\text {base }}\left(\mathbf{r}_{r}, \mathbf{r}_{s}\right) .
$$

Next, we estimate the baseline velocity model from the baseline data using standard tomographic techniques for a single data set. Third, we set up the linear forward problem,

$$
\mathbf{d}=\mathbf{G m} \text {. }
$$

The data vector with observed time-lapse traveltime delays is denoted $\mathbf{d}$, the modeling matrix is written as $\mathbf{G}$, and the model parameter vector is $\mathbf{m}$ (Tarantola, 1987; Menke, 1989). A grid parameterization can be used for the time-lapse velocity field.

In the final step, the estimated time-lapse velocity field $\Delta v_{4 \mathrm{D}}(\mathbf{r})$ is obtained by inverting the observed time-lapse traveltime shifts. The common least-squares inversion technique is used in the inversion step because the forward problem is linear. According to Tarantola (1987) and Menke (1989), the estimated model parameter vector $\mathbf{m}_{\text {est }}$ in the least-squares inversion is given by

$$
\mathbf{m}_{\mathrm{est}}=\left[\mathbf{G}^{t} \mathbf{C}_{D}^{-1} \mathbf{G}+\mathbf{C}_{M}^{-1}\right]^{-1} \mathbf{G}^{t} \mathbf{C}_{D}^{-1} \mathbf{d},
$$

with the data and model covariance matrix denoted as $\mathbf{C}_{D}$ and $\mathbf{C}_{M}$, respectively. We use $\mathbf{C}_{D}=\mathbf{I}$ and $\mathbf{C}_{M}=\sigma^{2} \mathbf{I}$, where $\sigma$ is an arbitrary constraint value.

\section{The linear forward modeling problem}

The linear forward modeling problem, where the 4D traveltime shift in equation 1 is computed, can be based on the well-known ray theory or on a finite-frequency wavefield theory. The ray theory is a solution of the wave equation in the high-frequency limit. According to Červený (2001), the reference traveltime for a wavefield in a heterogeneous reference velocity model $v_{\text {ref }}(\mathbf{r})$ is given by

$$
t_{\text {ref }}\left(\mathbf{r}_{r}, \mathbf{r}_{s}\right)=\int_{\mathbf{r}_{s}}^{\mathbf{r}_{r}} \frac{d r}{v_{\text {ref }}(r)},
$$

where the integration is performed along the raypath (i.e., the ray can be straight or bent) connecting the source and receiver. The perturbed traveltime for a wavefield with the same source and receiver positions as the reference wavefield is then

$t_{\text {pert }}\left(\mathbf{r}_{r}, \mathbf{r}_{s}\right)=\int_{\mathbf{r}_{s}}^{\mathbf{r}_{r}} \frac{d r}{v_{\text {pert }}(r)} \approx t_{\text {ref }}\left(\mathbf{r}_{r}, \mathbf{r}_{s}\right)-\int_{\mathbf{r}_{s}}^{\mathbf{r}_{r}} \frac{\Delta v(\mathbf{r})}{v_{\text {ref }}^{2}(\mathbf{r})} d r$,

with $\Delta v(\mathbf{r})$ being the velocity anomaly field and $v_{\text {pert }}(\mathbf{r})=v_{\text {ref }}(\mathbf{r})$ $+\Delta v(\mathbf{r})$ the perturbed velocity field.

This step is valid for a small perturbed velocity field compared to the reference velocity field. By subtracting equation 4 from equation 5 , one obtains that, according to the ray theory, the traveltime shift $\Delta t\left(\mathbf{r}_{r}, \mathbf{r}_{s}\right)=t_{\text {pert }}\left(\mathbf{r}_{r}, \mathbf{r}_{s}\right)-t_{\text {ref }}\left(\mathbf{r}_{r}, \mathbf{r}_{s}\right)$ is given by

$$
\Delta t\left(\mathbf{r}_{r}, \mathbf{r}_{s}\right) \approx-\int_{\mathbf{r}_{s}}^{\mathbf{r}_{r}} \frac{\Delta v(\mathbf{r})}{v_{\mathrm{ref}}^{2}(\mathbf{r})} d r
$$

Equation 6 is identical to equation 9 in Røste et al. (2006) (with $\alpha$ going to infinity), who estimate layer thickness and velocity changes from $4 \mathrm{D}$ prestack seismic data.

In a time-lapse mode, expression 6 is used again. The reference traveltime equals the baseline traveltime (i.e., $t_{\text {ref }}\left(\mathbf{r}_{r}, \mathbf{r}_{s}\right)$ $\left.=t_{\text {base }}\left(\mathbf{r}_{r}, \mathbf{r}_{s}\right)\right)$, and the perturbed traveltime is equal to the monitor traveltime (i.e., $t_{\text {pert }}\left(\mathbf{r}_{r}, \mathbf{r}_{s}\right)=t_{\text {mon }}\left(\mathbf{r}_{r}, \mathbf{r}_{s}\right)$ ). The time-lapse traveltime delay $\Delta t_{4 \mathrm{D}}\left(\mathbf{r}_{r}, \mathbf{r}_{s}\right)$ consequently is written as 


$$
\Delta t_{4 \mathrm{D}}\left(\mathbf{r}_{r}, \mathbf{r}_{s}\right) \approx-\int_{\mathbf{r}_{s}}^{\mathbf{r}_{r}} \frac{\Delta v_{4 \mathrm{D}}(\mathbf{r})}{v_{\text {base }}^{2}(\mathbf{r})} d r,
$$

where the velocity anomaly field is replaced by the $4 \mathrm{D}$ velocity field $\Delta v_{4 \mathrm{D}}(\mathbf{r})$. In a time-lapse inversion based on the ray theory, expression 7 is used to construct the linear forward problem.

In contrast to the high-frequency assumption in the ray theory, recordings of wavefields propagating through the subsurface exhibit finite-frequency properties. In a time-lapse mode, the finite-frequency traveltime delay $\Delta t_{4 \mathrm{D}}\left(\mathbf{r}_{r}, \mathbf{r}_{s}\right)=t_{\text {mon }}\left(\mathbf{r}_{r}, \mathbf{r}_{s}\right)-t_{\text {base }}\left(\mathbf{r}_{r}, \mathbf{r}_{s}\right)$ inherent in time-lapse velocity anomalies between the source and receiver is, to the first order of approximation, given by

$$
\Delta t_{4 \mathrm{D}}\left(\mathbf{r}_{r}, \mathbf{r}_{s}\right)=\int_{V} \Delta v_{4 \mathrm{D}}(\mathbf{r}) K_{\Delta t_{4 \mathrm{D}}}(\mathbf{r}) d \mathbf{r} .
$$

Here, $V$ is the target volume between the source and receiver, and $K_{\Delta t_{4 \mathrm{D}}}(\mathbf{r})$ is known as the finite-frequency sensitivity kernel or the Fréchet kernel for the 4D traveltime delay attribute.

Woodward (1992), Marquering et al. (1999), Hung et al. (2001), Spetzler and Snieder (2001, 2004), and Jocker et al. (2006) show how to take the finite-frequency characteristics of propagating wavefields into account for single data sets. For time-lapse data, the finite-frequency Fréchet kernel for a heterogeneous baseline model is derived in Appendix A. We find that

$$
\begin{aligned}
K_{\Delta t_{4 \mathrm{D}}}(\mathbf{r})= & -J\left(\mathbf{r}_{r}, \mathbf{r}_{s}\right) \int_{f_{0}-\Delta f}^{f_{0}+\Delta f} \frac{\mathcal{A}(f) \sqrt{f}}{v_{\text {base }}^{2.5}(\mathbf{r}) J\left(\mathbf{r}, \mathbf{r}_{s}\right) J\left(\mathbf{r}_{r}, \mathbf{r}\right)} \\
& \times \sin \left(2 \pi f\left[t\left(\mathbf{r}, \mathbf{r}_{s}\right)+t\left(\mathbf{r}_{r}, \mathbf{r}\right)-t\left(\mathbf{r}_{r}, \mathbf{r}_{s}\right)\right]\right. \\
& \left.+\frac{\pi}{4}\right) d f,
\end{aligned}
$$

where $f$ stands for frequency.

Figure 1 illustrates the process of integration over the target zone between the source and receiver positions. The geometric spreading factor $J$ and the traveltime $t$ can be calculated from a homogeneous as well as a heterogeneous baseline medium (i.e., ray-bending effects are included). The ballistic wavefield joining the source and receiver have the traveltime $t\left(\mathbf{r}_{r}, \mathbf{r}_{s}\right)$ and geometric spreading factor $J\left(\mathbf{r}_{r}, \mathbf{r}_{s}\right)$. For the detour wavefield from the source position via the integration point $\mathbf{r}$ (i.e., a single scattering point) back to the receiver position, the traveltime and geometric spreading factor from the source position to the integration point are $t\left(\mathbf{r}, \mathbf{r}_{s}\right)$ and $J\left(\mathbf{r}, \mathbf{r}_{s}\right)$; the traveltime and geometric spreading factor from the integration point to the receiver position are $t\left(\mathbf{r}_{r}, \mathbf{r}\right)$ and $J\left(\mathbf{r}_{r}, \mathbf{r}\right)$.

The finite-frequency sensitivity kernel in equation 9 is integrated over the frequency band $\left[f_{0}-\Delta f ; f_{0}+\Delta f\right]$ to account for the

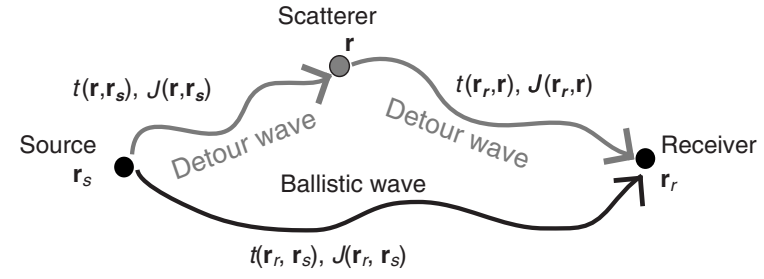

Figure 1. Schematic of the integration process over the target zone between source and receiver wells. broadband frequency properties of propagating finite-frequency waves. The amplitude spectrum of a recorded wavefield is written as $\mathcal{A}(f)$. A normalization condition is applied to the amplitude spectrum so that $\int_{f_{0}-\Delta f}^{f_{0}+\Delta f} \mathcal{A}(f) d f=1$ in order to obtain the proper broadband sensitivity function. To compute the Fréchet kernel $K_{\Delta t}(\mathbf{r})$, one must know the source and receiver positions, an appropriate reference velocity model, and the power spectrum of the recorded wavefield. The first two requirements are also needed in the ray theory; the last point can be obtained from the observed wavefields.

Figure 2 shows examples of time-lapse, finite-frequency sensitivity kernels and raypaths for the crosswell acquisition setup (see Figure 3) used in this paper. The heterogeneous baseline velocity model used for the Fréchet kernel and raypath computations is shown in Figure 4a. Notice that the sensitivity kernels have the most sensitivity in a limited area around the raypaths. This area is the first Fresnel volume. In addition, ray-bending effects inherent to the heterogeneous reference velocity model are included in the 4D raypaths and the Fréchet kernels.

The importance of the finite-frequency Fréchet kernel for traveltime delays in equation 9 is verified experimentally by Hung et al. (2001) and Jocker et al. (2006). In brief, these workers have an experimental setup wherein a spherical anomaly in a homogeneous reference medium scatters an incoming wavefield, which then is perturbed. Hung et al. (2001) apply a numerical method to simulate 3D wavefields for the reference and perturbed medium, respectively. These wavefields are then used to test the validity of the finite-frequency sensitivity kernels for traveltime shifts. Jocker et al. (2006) do not limit the validation experiment to numerical wavefield simulations, but they apply real 3D ultrasonic waveform recordings.

The linear finite-frequency wave theory is a natural extension of the ray theory. One can show that in the high-frequency limit, the 4D time delays in equation 8 are equal to the time shifts computed with the ray theory in equation 7 . Hence,

$$
\lim _{f \rightarrow \infty} \Delta t_{4 \mathrm{D}}\left(\mathbf{r}_{r}, \mathbf{r}_{s}\right) \approx-\int_{\mathbf{r}_{s}}^{\mathbf{r}_{r}} \frac{\Delta v_{4 \mathrm{D}}(\mathbf{r})}{v_{\text {base }}^{2}(\mathbf{r})} d r,
$$

where the integration of the velocity perturbation field or time-lapse velocity field is along the raypath connecting the source and receiv-

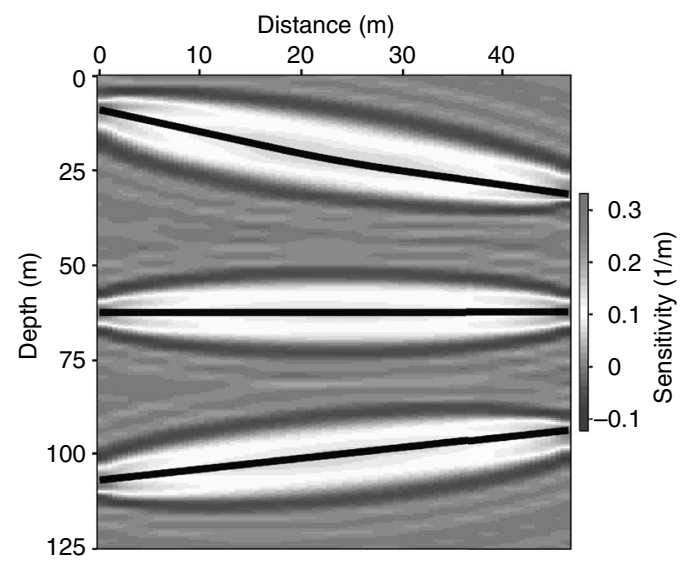

Figure 2. Sensitivity kernels for the time-lapse traveltime delay and the associated raypaths. The raypaths are indicated by solid black lines; the shaded bar shows the finite-frequency sensitivity to the time-lapse velocity field. 
er. Spetzler and Snieder $(2001,2004)$ and Jocker et al. (2006) derive expression 10 .

The validity of the ray theory (Červený, 2001) is limited to media with anomalies of a size $a$ larger than the wavelength and the width $L_{F}$ of the first Fresnel volume (from now on called the Fresnel volume); hence,

$$
\frac{\lambda}{a}<1 \quad \text { and } \frac{L_{F}}{a}<1
$$

On the other hand, the validity of the finite-frequency wave theory extends to media with velocity anomalies smaller than the Fresnel volume width, i.e.,

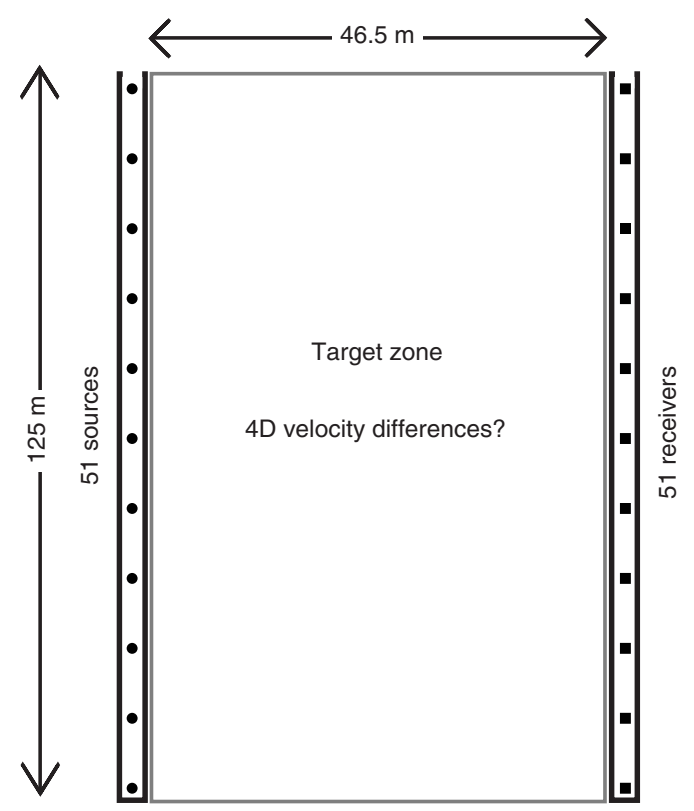

Figure 3. Schematic of the acquisition setup of the Durham ultrasonic wave experiment.

a) $0 \quad \begin{array}{cc}\text { Distance }(\mathrm{m}) \\ 20 & 30\end{array}$

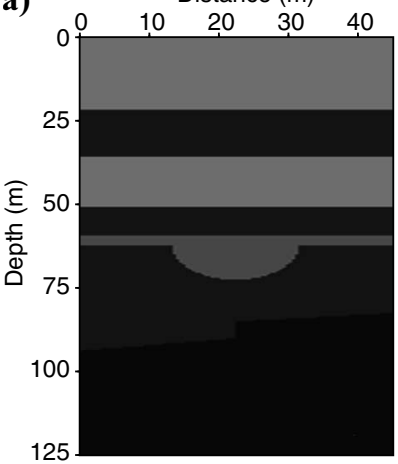

b) Distance $(\mathrm{m})$

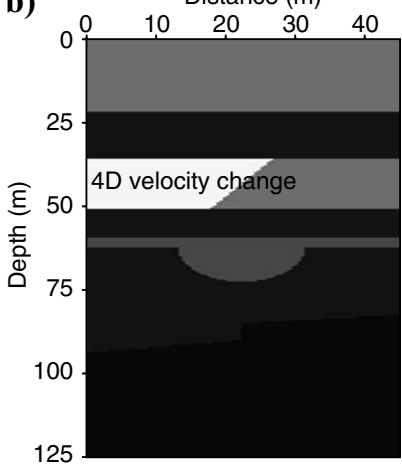

$220024002600 \quad 2800 \quad 3000$

Velocity $(\mathrm{m} / \mathrm{s})$

Figure 4. (a) The baseline velocity model (preflooding) and (b) the monitor velocity model (postflooding).

$$
\frac{L_{F}}{a}>1
$$

The Fresnel volume $L_{F}=\sqrt{\lambda L}$ ( $\lambda$ is the wavelength and $L$ is the raypath length) is the area between the source and receiver for which the scattered wavefields contribute constructively at the receiver position (e.g., Spetzler and Snieder, 2001, 2004).

\section{METHOD VALIDATION BY 3D REAL ULTRASONIC WAVEFORM DATA}

To validate the time-lapse monitoring method for $3 \mathrm{D}$ wavefield propagation, we work with ultrasonic waveform data from the Durham laboratory experiment (Legget et al., 1993; Pratt, 1999). In this experiment, a crosswell seismic acquisition geometry with two parallel wells was simulated (see Figure 3 ). There are 51 source positions (indicated with black circles) at one side of the target zone and 51 receiver positions (plotted with black squares) at the opposite side. Two physical models consisting of seven layers with five different epoxy resin mixtures (i.e., five different velocities) form the time-lapse model, with the baseline and monitor structure in pre- and postflooding situations, respectively.

The baseline and monitor models (after rescaling) are shown in Figure $4 \mathrm{a}$ and $\mathrm{b}$, respectively. Notice the geologic features such as the channel feature and fault structure (at a 90-m depth) in both models. The time-lapse velocity difference (i.e., about $-400 \mathrm{~m} / \mathrm{s}$ ) between the baseline and monitor structure is located in layer three from the top. The scaled baseline and monitor models were made from the same solid block in the same mold. To incorporate the 4D difference between the baseline and monitor model, a "flooding area" was cut out by machine in half of the block, which was then covered by a different epoxy mix to form the postflood zone. The other half of the block was left intact to simulate the preflooding situation. Finally, the complete block was cut in two: 1) the preflood model and 2) the postflood model. Except for the time-lapse differences between the baseline and monitor models, the two blocks are assumed to be identical within the errors of the cutting machine (i.e., $0.025 \mathrm{~mm}$ ).

To generate and record ultrasonic waveform data, $500-\mathrm{kHz}$ transducers were used. The source transducer has a circular cross section of $7 \mathrm{~mm}$ diameter; the cross-section diameter of the receiver transducer is $1 \mathrm{~mm}$. The source and receiver spacing is $2.5 \mathrm{~mm}$. Each block was submerged in a water tank. Then the ultrasonic transducers were placed on each side to simulate the crosswell geometry with parallel wells, as shown in Figure 3. Proper care was taken to repeat the baseline source and receiver positions in the monitor crosswell survey to avoid problems with nonrepeatability effects in the timelapse monitoring experiment. However, smaller errors in the sourcereceiver geometry between the baseline and monitor surveys do occur, resulting in traveltime shifts where none are expected.

The ultrasonic baseline and monitor data sets consist of 2601 data points each (i.e., 51 sources times 51 receivers). The bandwidth of the ultrasonic 4D waveform data is between 200 and $600 \mathrm{kHz}$, and the dimensions of the scaled baseline and monitor models are 46.5 $\times 125 \mathrm{~mm}$. The seismic-to-sonic dispersion is negligible, so upscaling of the physical model is allowed. A scaling factor of 1000 is used to simulate realistic dimensions of a crosswell survey. Hence, the simulated well separation is $46.5 \mathrm{~m}$, the distance between the top and bottom source-receiver is $125 \mathrm{~m}$, and the simulated seismic bandwidth is $200-600 \mathrm{~Hz}$. 
Shot gathers (after rescaling the traveltime) for the source position located in the layer with time-lapse differences (the source depth is $42.5 \mathrm{~m}$ ) from the baseline and monitor survey are shown in Figure $5 \mathrm{a}$ and $\mathrm{b}$. The third top layer has a lower velocity compared to the surrounding layers. This is seen clearly in the baseline and especially in the monitor common-shot gather because the waveform at sourcedepth level is significantly delayed. The postflooding zone is about $400 \mathrm{~m} / \mathrm{s}$ slower than the preflooding zone; hence, the waveforms in the monitor common-shot gather are even more distorted by a developing triplication (i.e., the half-bowtie; Kravtsov, 1988). The difference wavefield between the baseline and monitor common-shot gather is shown in Figure 5c. As expected, the largest differences in the time-lapse data are observed for the receiver positions located in the third top layer (i.e., between 35 and 50-m depth) with the flooding zone.

To estimate the time-lapse traveltime delay between the observed baseline and monitor data, we used the following processing scheme:

1) A time window (between the two white lines) around the firstarriving wavefields was defined for the baseline and monitor data; in Figure 5, the time window around the first arrival is indicated.

2) The wave energy outside the time window was set to zero.

3) The time-windowed first arrivals were band-pass-filtered between 200 and $600 \mathrm{~Hz}$ (i.e., the seismic bandwidth with the highest signal-to-noise level).

4) The 4D traveltime shifts between the processed baseline and monitor data were estimated by crosscorrelation. The timelapse traveltime delay $\Delta t_{4 \mathrm{D}}$ was measured at the maximum value of the crosscorrelation integral of the baseline data $d_{\text {base }}(t)$ and monitor data $d_{\text {mon }}(t)$. Hence, $\Delta t_{4 \mathrm{D}}$ was measured at maximum value of

$$
\int_{-\infty}^{\infty} d_{\text {base }}\left(\Delta t_{4 \mathrm{D}}-t\right) d_{\mathrm{mon}}(t) d t .
$$

5) The measured 4D traveltime shifts from expression 13 were used as input data in the time-lapse tomographic monitoring method.

The structure of the baseline and monitor model is $2.5 \mathrm{D}$ because of the constant-velocity structure perpendicular to the source- a) Receiver position (m)
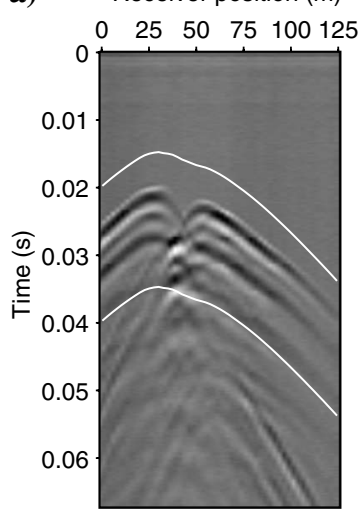

b) Receiver position $(\mathrm{m})$

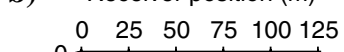

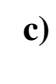

c) Receiver position $(\mathrm{m})$

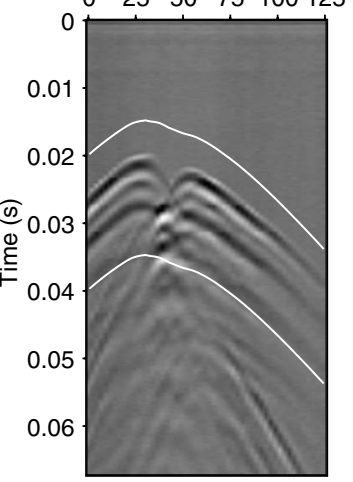

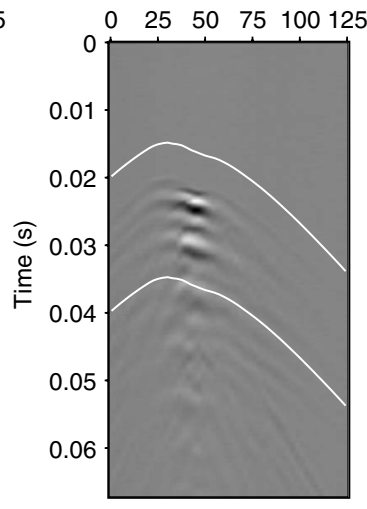

Figure 5. Shot gathers in the real ultrasonic waveform experiment for the source position at $42.5-\mathrm{m}$ depth. The time window for the first arrival is between the two solid white lines. (a) The baseline shot gather, (b) the monitor shot gather, and (c) the difference shot gather. receiver cross section. In turn, the finite-frequency wavefield theory in equation 8 for $2 \mathrm{D}$ wave propagation is applicable. The finite-frequency sensitivity kernel for each source-receiver pair is therefore calculated only in the plane with the source-receiver cross section. Appendix B shows that a 2.5D model is equal to a $2 \mathrm{D}$ medium for the finite-frequency wavefield theory.

For the ray theory, we apply equation 7 , in which the raypaths are computed in the source-receiver cross section. The conditions for both the ray theory and the finite-frequency wavefield theory are satisfied for the flooding zone in layer three because, according to equation 11, the vertical length of the time-lapse anomaly is comparable to the Fresnel volume width. In other words, the wavelength $\lambda$ $=5 \mathrm{~m}$ and the well separation $L=46.5 \mathrm{~m}$, which results in $L_{F}$ $=\sqrt{\lambda L} \approx 15 \mathrm{~m}$ for the Fresnel volume. The thickness of layer three is likewise about $a=15 \mathrm{~m}$; hence, $L_{F} / A \approx 1$.

With the observed time-lapse traveltime delays from the baseline and monitor data and the forward problem in equation 2 based on either the ray theory or the finite-frequency wavefield theory, the inversion problem in equation 3 was solved. The target zone between the parallel source and receiver well as shown in Figure 3 was parameterized using a grid with $24 \times 64$ elements in the horizontal and vertical directions, respectively. A smoothing factor $\sigma$ through $\mathbf{C}_{M}^{-1}$ $=\sigma^{2} \mathbf{I}$ (known as the Tikhonov regularization; Hansen, 1998) in the inverse problem in equation 3 was required to stabilize the $4 \mathrm{D}$ tomographic inversion. The value of the regularization factor is chosen as small as possible to reduce the effect of smoothing on resolving the inverted time-lapse velocity field.

The estimated time-lapse models compiled with the finite-frequency wavefield theory and the ray theory are shown in Figure 6a and $b$, respectively. The two estimated 4D models locate the flooding zone at the correct position in layer three (for a comparison, see Figure $4 \mathrm{~b}$ with the monitor velocity model). The inverted time-lapse velocity model compiled with the finite-frequency wavefield theory has soft edges at the sides of the flooding zone, whereas the estimated 4D model from the ray theory tends to have sharp edges at the flooding-zone boundaries. Both estimated models have dipping stripes on the right side, which is an example of the common tomographic problem of nonuniform raypath coverage (see Figure 6 in Legget et al., 1993). Consequently, nonuniform raypath coverage may bias the inverted model. In inversion theory, this is known as spectral leakage (Trampert and Snieder, 1996). The estimated timelapse velocity models in Figure 6 are similar to the inverted 4D structure in Legget et al. (1993, Figure 10c).

\section{D SYNTHETIC VALIDATION EXPERIMENT}

The 3D ultrasonic waveform experiment is supplemented by a 2D synthetic finite-difference modeling experiment to put the time-lapse tomographic monitoring method to an additional validation test. The finite-difference simulation of the time-lapse crosswell experiment is identical to the ultrasonic waveform experiment, except for the dimensionality of the wave-propagation problem (two instead of three dimensions). The acquisition geometry of the crosswell experiment is illustrated in Figure 3; the baseline and monitor $\mathrm{P}$-wave velocity structures of the target zone are 
plotted in Figure 4a and b, respectively. The P-wave structure is repeated in the S-wave velocity baseline and monitor model, wherein the ratio between the P-wave velocity and S-wave velocity equals $\sqrt{3}$. A constant-density model with the density equal to $2766 \mathrm{~kg} / \mathrm{m}^{3}$ is applied in the baseline and the monitor medium.

To compute the time-lapse crosswell data, a 2D full elastic scheme (Robertson et al., 1994; van Vossen et al., 2002) was used. This numerical wavefield-modeling method uses a staggered grid with second-order accuracy in time and fourth-order accuracy in space (i.e., $O(2,4))$. Proper care was taken to satisfy the Courant stability criterion. The source function is a Ricker wavelet that had a broad bandwidth with the central frequency fixed to $500 \mathrm{~Hz}$. To avoid reflections from the model boundaries, extra grid cells, including an absorbing taper, were implemented on all four sides of the target zone. Last, noise with a signal-to-noise level equal to three was added to the synthetic baseline and monitor data.

The synthetic baseline, monitor, and difference common-shot gather for the source depth at $42.5 \mathrm{~m}$ are shown in Figure 7. Because of the low signal-to-noise level, the triplication resulting from the low-velocity zone is difficult to see in the shot gathers. The processing scheme described in the section about the Durham ultrasonic time-lapse experiment was used on the synthetic time-lapse data. Thereby, the traveltime delays between the baseline and monitor wavefields were measured and then used in the inversion to obtain the estimated time-lapse models compiled with either the ray theory or the finite-frequency wavefield theory.

The inverted time-lapse models are presented in Figure 8. The flooding zone estimated by time-lapse tomography in the synthetic experiment is located correctly, according to the true time-lapse model in Figure 4. A comparison of Figures 6 and 8 reveals many similarities between the estimated time-lapse model in the real and synthetic validation tests. Nonuniform raypath coverage is again responsible for the dipping stripes on the right-hand side of the flooding zone.

\section{DISCUSSION}

The common crosswell tomographic method is extended to a time-lapse mode, where traveltime differences between the baseline

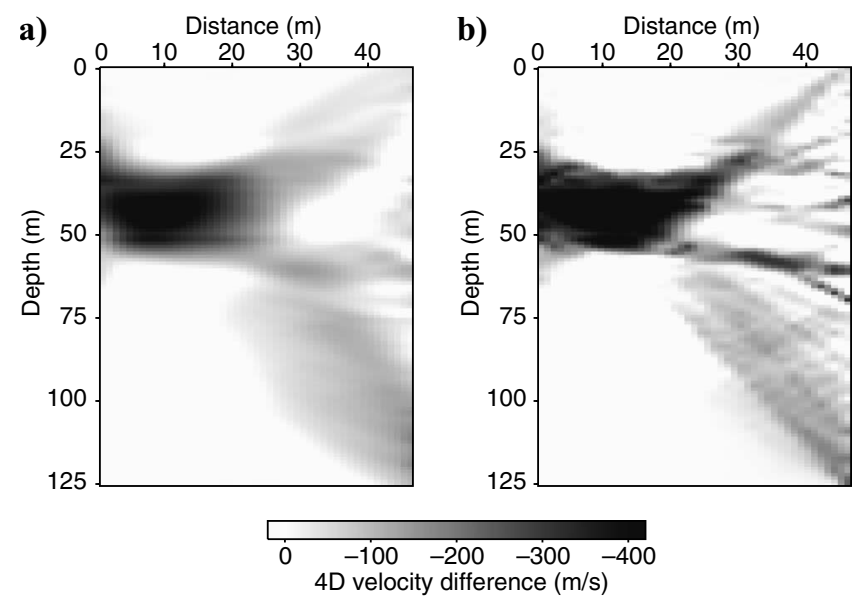

Figure 6. The estimated time-lapse velocity field in the real Durham flooding simulation experiment. (a) The time-lapse model compiled with the finite-frequency wave theory: (b) The time-lapse velocity model from the ray theory. and monitor arrivals are used to estimate the change in velocity structure between the source and receiver wells. To improve the resolution of inverted time-lapse velocity structures smaller than the Fresnel volume, a linear finite-frequency wave theory instead of the standard ray theory was implemented in the tomographic method. In this specific modeling experiment, the time-lapse velocity structure simulating a flooding zone in the epoxy model was comparable in size to the Fresnel volume of the ultrasonic waveform data. Consequently, the ray theory worked just as well as the linear finite-frequency wave theory. On the other hand, if one would consider the epoxy model for the baseline structure or the monitor structure in Figure 4 , the advantage of using the finite-frequency wave theory becomes clear. Specifically, the two models have one channel-like anomaly that is smaller than the first Fresnel zone. Jocker et al. (2006, Figure 12) show an example using the same ultrasonic data, demonstrating that the finite-frequency wave theory is more adequate than the ray theory to image structures smaller than the Fresnel volume.

Another important problem in crosswell tomography is artifacts in the estimated model inherent to nonuniform raypath coverage (also known as spectral leakage). To some extent, it is possible to reduce or solve the spectral leakage problem, which in global surfacewave tomography is done by Trampert and Snieder (1996) and Spet- a)

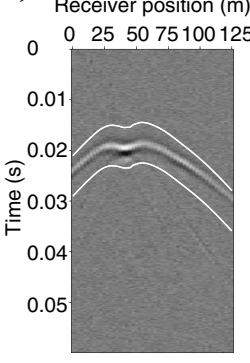

b)

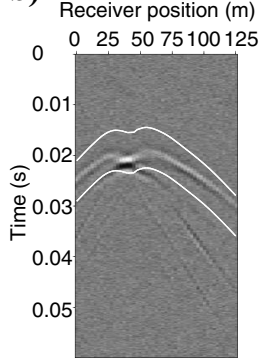

c)

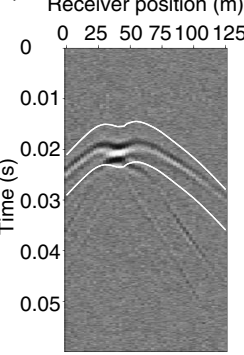

Figure 7. Shot gathers in the synthetic modeling experiment. The source position is at 42.5-m depth. The time window for the first arrival is plotted between the two solid white lines. (a) The baseline shot gather, (b) the monitor shot gather, and (c) the difference shot gather.

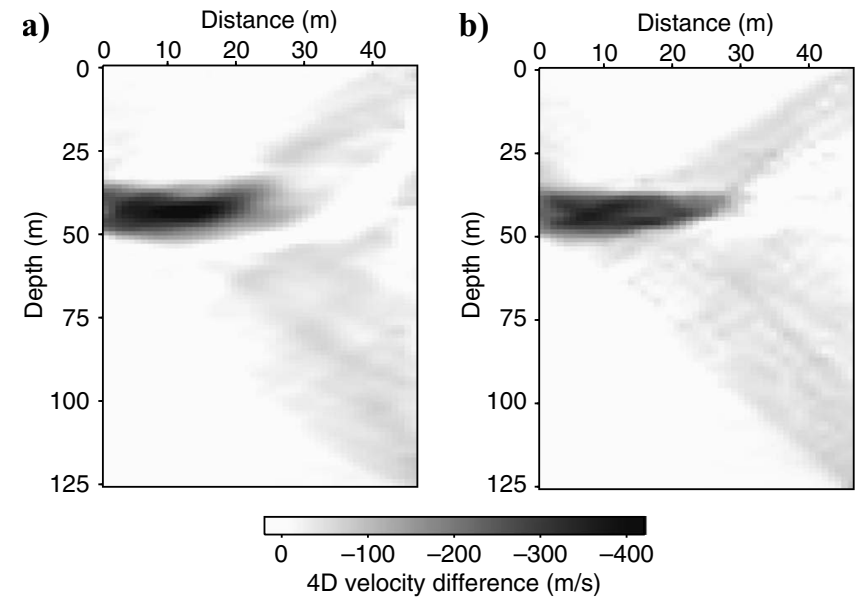

Figure 8. The estimated time-lapse velocity field in the synthetic modeling experiment. (a) The inverted time-lapse velocity model from the finite-frequency wave theory: (b) The inverted time-lapse velocity model using the ray theory. 
zler and Trampert (2003). The technique of Trampert and Snieder (1996) (where spherical harmonic functions are used to express the phase velocity field) can be applied in crosswell tomography, where the basis functions are 2D sine functions instead. However, such an approach would only work well for a single anomaly because the 2D sine functions are global basis functions (e.g., Spetzler, 2006). To account for multiple anomalies, one could try an inversion using local basis functions such as wavelets in the series expansion of the timelapse velocity field.

In any case, it is beyond the scope of this paper to investigate the complex problem with nonuniform raypath coverage further. Other alternatives to reduce the effect of nonuniform raypath coverage could be to use a staggered grid (Vesnaver and Bohm, 2000) or fullwaveform inversion techniques (Pratt, 1999; Gao et al., 2003). However, the success of the latter method depends on how accurately the source wavelet is estimated. The developed time-lapse tomographic method uses the traveltime difference between the baseline and monitor waveforms, which can be estimated from crosscorrelation without knowing the source wavelet.

Our time-lapse tomographic method, based on the finite-frequency wave theory, is limited to a 3D ultrasonic wave laboratory experiment and a 2D synthetic finite-difference modeling experiment. For an example of applying the finite-frequency wave theory on real data, we refer to Spetzler et al. (2006), who use 4D crosswell tomography to monitor injected $\mathrm{CO}_{2}$. In addition, for time-lapse experiments with a good control of the source mechanism, it is possible to perform a time-lapse monitoring evaluation by tomography using the amplitude attribute differences between the baseline and monitor data. For instance, Spetzler (2006) uses 4D amplitude tomography to image the time-lapse structure of a heavy oil reservoir in which steam had been injected to heat the tar sand formation.

\section{CONCLUSIONS}

The finite-frequency effect of propagating waves can be implemented in time-lapse tomography. Thereby, it is possible to perform tomography in media with time-lapse structures smaller than the Fresnel volume. In contrast, a tomographic method based on the ray theory is limited to anomalies larger than the Fresnel volume.

We suggest validating seismic imaging methods using real ultrasonic measurements whenever possible. Real waveform data do not suffer from approximations and assumptions that are often made in numerical wavefield-simulation methods (e.g., plane-wavefield propagation, the high-frequency approximation in the ray theory, or hybrid approaches.)

The developed time-lapse tomographic approach is not limited to crosswell settings. One needs information about the source and receiver geometry; in addition, a baseline velocity model is required, and the frequency range with the highest signal-to-noise ratio must be estimated. Those three requirements are available in vertical seismic profiling (VSP) experiments; hence the 4D methodology is certainly applicable to time-lapse VSP data.

\section{ACKNOWLEDGMENTS}

This work was sponsored by the Dutch technology organization STW, project DAR.6293, and through the CATO project. We gratefully acknowledge Neil Goulty and Gerhard Pratt for permission to use the Durham laboratory data. Associate editor Johan Robertsson and three reviewers (Ivan Vasconcelos, Aldo Vesnaver, and an anon- ymous reviewer) provided good comments that helped us to improve the quality of the paper. We intend to use the developed 4D methodology in the EU-funded RECOPOL project (acronym for Reduction of $\mathrm{CO}_{2}$ emission by means of $\mathrm{CO}_{2}$ storage in coal seams in the Silesian coal basin of Poland).

\section{APPENDIX A}

\section{DERIVATION OF THE FINITE-FREQUENCY KERNEL FOR A HETEROGENEOUS VELOCITY REFERENCE MODEL}

The first-order Rytov approximation (Aki and Richards, 1980; Woodward, 1992; Snieder and Lomax, 1996; Spetzler and Snieder, 2001 ) is used to derive the $2 \mathrm{D}$ finite-frequency wavefield theory for time-lapse traveltime delays. The Rytov approximation only describes contrasts in P-wave velocity (or slowness equal to the inverse of velocity) but neglects any density contrast and shear-wave effects. Slowness is used instead of velocity in the derivation. In the final expression for the finite-frequency sensitivity kernel for time-lapse traveltime delays, slowness is replaced by velocity again.

The Rytov wavefield $P_{R}\left(\mathbf{r}_{r}, \mathbf{r}_{s}, \omega\right)$ (i.e., the monitor wavefield) at the angular frequency $\omega=2 \pi f$ (with $f$ denoting the frequency in hertz) emitted from the source position $\mathbf{r}_{s}$ and recorded at the receiver position $\mathbf{r}_{r}$ is given by

$$
P_{R}\left(\mathbf{r}_{r}, \mathbf{r}_{s}, \omega\right)=P_{\text {base }}\left(\mathbf{r}_{r}, \mathbf{r}_{s}, \omega\right) \exp \left(\frac{P_{B}}{P_{\text {base }}}\left(\mathbf{r}_{r}, \mathbf{r}_{s}, \omega\right)\right),
$$

which accounts for the single-scattering process of a propagating wavefield in heterogeneous media (see Figure 1). The baseline wavefield inherent in the arbitrary baseline slowness field $u_{\text {base }}(\mathbf{r})$ is denoted $P_{\text {base }}\left(\mathbf{r}_{r}, \mathbf{r}_{s}, \omega\right)$, and the first-order Born wavefield is written as $P_{B}\left(\mathbf{r}_{r}, \mathbf{r}_{s}, \omega\right)$.

The monochromatic time-lapse phase perturbation $\Delta \varphi_{4 \mathrm{D}}\left(\mathbf{r}_{r}\right.$, $\left.\mathbf{r}_{s}, \omega\right)$ of the scattered field inherent in the time-lapse slowness perturbation field $\Delta u_{4 \mathrm{D}}(\mathbf{r})$ in the arbitrary baseline slowness field $u_{\text {base }}(\mathbf{r})$ is obtained from the imaginary part of the exponential function in equation A-1. Hence, the time-lapse phase delay at angular frequency $\omega$ is given by

$$
\Delta \varphi_{4 \mathrm{D}}\left(\mathbf{r}_{r}, \mathbf{r}_{s}, \omega\right)=\Im\left(\frac{P_{B}}{P_{\text {base }}}\left(\mathbf{r}_{r}, \mathbf{r}_{s}, \omega\right)\right) .
$$

The time-lapse traveltime residual $\Delta t_{4 \mathrm{D}}$ is then equal to

$$
\Delta t_{4 \mathrm{D}}\left(\mathbf{r}_{r}, \mathbf{r}_{s}, \omega\right)=\frac{1}{\omega} \mathfrak{I}\left(\frac{P_{B}}{P_{\text {base }}}\left(\mathbf{r}_{r}, \mathbf{r}_{s}, \omega\right)\right),
$$

where the linear expression $\Delta \varphi_{4 \mathrm{D}}=\omega \Delta t_{4 \mathrm{D}}$ is used.

In the Durham experiment, the baseline slowness field is heterogeneous, which the Born first wavefield (from now on called the Born wavefield) takes into account. The monochromatic Born wavefield for a point source in a heterogeneous velocity model with a constant density is given by

$$
\begin{aligned}
P_{B}\left(\mathbf{r}_{r}, \mathbf{r}_{s}, \omega\right)= & -2 \omega^{2} \int_{\mathbf{V}} u_{\text {base }}(\mathbf{r}) P_{\text {base }}\left(\mathbf{r}, \mathbf{r}_{s}\right) \\
& \times \Delta u_{4 \mathrm{D}}(\mathbf{r}) G\left(\mathbf{r}_{r}, \mathbf{r}\right) d V
\end{aligned}
$$

(Aki and Richards, 1980; Woodward, 1992; Snieder and Lomax, 
1996; Spetzler and Snieder, 2001). The (2D) volume integration is indicated by $\mathbf{V}$. The time-lapse slowness contrast $\Delta u_{4 D}(\mathbf{r})$ in equation A-4 acts as a scattering term (e.g., the scatterer in Figure 1). The 2D far-field monochromatic point source Green's function $G\left(\mathbf{r}_{r}, \mathbf{r}\right)$ for a heterogeneous reference (baseline) slowness field can be found in Snieder and Chapman (1998); thus,

$$
\begin{aligned}
G\left(\mathbf{r}_{r}, \mathbf{r}, \omega\right)= & -\frac{1}{\sqrt{8 \pi \omega u_{\mathrm{base}}(\mathbf{r})} J\left(\mathbf{r}_{r}, \mathbf{r}\right)} \\
& \times \exp i\left(\omega t\left(\mathbf{r}_{r}, \mathbf{r}\right)+\frac{\pi}{4}\right) .
\end{aligned}
$$

The traveltime and geometric spreading factor for a wavefield from the scatterer $\mathbf{r}$ to the receiver $\mathbf{r}_{r}$ is denoted $t\left(\mathbf{r}_{r}, \mathbf{r}\right)$ and $J\left(\mathbf{r}_{r}, \mathbf{r}\right)$, respectively. Likewise, the initial wavefield from the point source to the scatterer in a heterogeneous baseline slowness field is equal to

$$
\begin{aligned}
P_{\text {base }}\left(\mathbf{r}, \mathbf{r}_{s}, \omega\right)= & -\frac{1}{\sqrt{8 \pi \omega u_{\text {base }}\left(\mathbf{r}_{s}\right)} J\left(\mathbf{r}, \mathbf{r}_{s}\right)} \\
& \times \exp i\left(\omega t\left(\mathbf{r}, \mathbf{r}_{s}\right)+\frac{\pi}{4}\right) .
\end{aligned}
$$

The traveltime and geometric spreading factors are written as $t\left(\mathbf{r}, \mathbf{r}_{s}\right)$ and $J\left(\mathbf{r}, \mathbf{r}_{s}\right)$, respectively. In addition, the ballistic wavefield $P_{\text {base }}\left(\mathbf{r}_{r}, \mathbf{r}_{s}, \omega\right)$ (see Figure 1) in equation A-1 from the source position $\mathbf{r}_{s}$ to the receiver position $\mathbf{r}_{r}$ is

$$
\begin{aligned}
P_{\text {base }}\left(\mathbf{r}_{r}, \mathbf{r}_{s}, \omega\right)= & -\frac{1}{\sqrt{8 \pi \omega u_{\text {base }}\left(\mathbf{r}_{s}\right)} J\left(\mathbf{r}_{r}, \mathbf{r}_{s}\right)} \\
& \times \exp i\left(\omega t\left(\mathbf{r}_{r}, \mathbf{r}_{s}\right)+\frac{\pi}{4}\right),
\end{aligned}
$$

where $t\left(\mathbf{r}_{r}, \mathbf{r}_{s}\right)$ and $J\left(\mathbf{r}_{r}, \mathbf{r}_{s}\right)$ are the traveltime and geometric spreading factors, respectively. Notice that equations A-5-A-7 are valid in the far-field because a paraxial approximation has been used implicitly (Spetzler and Snieder, 2004). Consequently, these three expressions become increasingly more accurate away from the source and receiver positions.

The Green's function in equations A-5 and A- 6 is inserted in the Born wavefield in expression A-4. Next, the Born wavefield and the ballistic pressure wavefield are inserted in equation A-3 to obtain the time-lapse traveltime delay at single angular frequency. The frequency-averaged time-lapse traveltime shift is obtained from

$$
\Delta t_{4 \mathrm{D}}\left(\mathbf{r}_{r}, \mathbf{r}_{s}\right)=\int_{\omega_{0}-\Delta \omega}^{\omega_{0}+\Delta \omega} A(\omega) \Delta t_{4 \mathrm{D}}\left(\mathbf{r}_{r}, \mathbf{r}_{s}, \omega\right) d \omega,
$$

where $A(\omega)$ is the normalized frequency-dependent amplitude spectrum from $\int_{\omega_{0}-\Delta \omega}^{\omega_{0}+\Delta \omega} A(\omega) d \omega=1$. After a thorough derivation and after replacing slowness with velocity, one can show that the frequencyaveraged time-lapse traveltime shift is given by

$$
\Delta t_{4 \mathrm{D}}\left(\mathbf{r}_{r}, \mathbf{r}_{s}\right)=\int_{\mathbf{V}} \Delta v_{4 \mathrm{D}}(\mathbf{r}) K_{d t_{4 \mathrm{D}}}(\mathbf{r}) d V,
$$

where $K_{d t_{4 \mathrm{D}}}(\mathbf{r})$ is the Fréchet kernel for the time-lapse traveltime delay attribute. In terms of traveltimes $t$ and geometric spreading factor
$J$, which need to be computed for every integration point $\mathbf{r}$, the finitefrequency Fréchet kernel is given by

$$
\begin{aligned}
K_{\Delta t_{4 \mathrm{D}}}(\mathbf{r})= & -J\left(\mathbf{r}_{r}, \mathbf{r}_{s}\right) \int_{f_{0}-\Delta f}^{f_{0}+\Delta f} \frac{\mathcal{A}(f) \sqrt{f}}{v_{\text {base }}^{2.5}(\mathbf{r}) J\left(\mathbf{r}, \mathbf{r}_{s}\right) J\left(\mathbf{r}_{r}, \mathbf{r}\right)} \\
& \times \sin \left(2 \pi f\left[t\left(\mathbf{r}, \mathbf{r}_{s}\right)+t\left(\mathbf{r}_{r}, \mathbf{r}\right)-t\left(\mathbf{r}_{r}, \mathbf{r}_{s}\right)\right]\right. \\
& \left.+\frac{\pi}{4}\right) d f .
\end{aligned}
$$

\section{APPENDIX B 2.5D EQUALS 2D FOR FINITE-FREQUENCY WAVEFIELD THEORY}

The frequency-averaged time-lapse traveltime shift expression for transmitted wavefields in 3D heterogeneous media is derived similarly to the $2 \mathrm{D}$ expression in Appendix A. However, the epoxy models in the Durham time-lapse experiment are $2.5 \mathrm{D}$ because the direction perpendicular to the source-receiver cross section of the velocity structure is laterally invariant. In that case, one can show, using the stationary-phase approximation (Bleistein, 1984), that 3D finite-frequency wave theory (and the Green's function) converges to the $2 \mathrm{D}$ case. The short version of the derivation is to use the fact that for the Green's function in a 3D medium with the lateral invariant structure in the $z$-axis direction,

$$
G^{2 \mathrm{D}}(x, y)=\int_{-\infty}^{\infty} G^{3 \mathrm{D}}(x, y, z) d z
$$

(Snieder, 2001)

\section{REFERENCES}

Aki, K., and P. G. Richards, 1980, Quantitative seismology, theory and methods: W. H. Freeman \& Co.

Bertrand, A., and C. MacBeth, 2003, Seawater velocity variations and realtime reservoir monitoring: The Leading Edge, 22, 351-355.

Billette, F., S. Le Bégat, P. Podvin, and G. Lambaré, 2003, Practical aspects and applications of 2D stereotomography: Geophysics, 68, 1008-1021.

Bleistein, N., 1984, Mathematical methods for wave phenomena: Academic Press Inc.

Boyd-Gorst, J., P. Fail, and L. Pointing, 2001, 4-D time lapse reservoir monitoring of Nelson field, central North Sea: Successful use of an integrated rock physics model to predict and track reservoir production: The Leading Edge, 20, 336-350.

Červený, V., 2001, Seismic ray tracing: Cambridge University Press.

Gao, F., A. Levander, R. G. Pratt, C. Zelt, and G. Fradelizio, 2003, Waveform tomography at a groundwater contamination site: VSP-surface data set: Geophysics, 71, no. 1, H1-H11.

Guilbot, J., and B. Smith, 2002, 4D constrained depth conversion for reservoir compaction estimation: Application to Ekofisk field: The Leading Edge, 21, 302-308.

Hansen, P. C., 1998, Rank-deficient and discrete ill-posed problems: Numerical aspects of linear inversion: SIAM

Hung, S., F. A. Dahlen, and G. Nolet, 2001, Wavefront healing: A bananadoughnut perspective: Geophysical Journal International, 146, 289-312.

Jocker, J., J. Spetzler, D. Smeulders, and J. Trampert, 2006, Validation of first-order wave diffraction theory by an ultrasonic experiment: Geophysics, 71, no. 6, T169-T177.

Kravtsov, Y. A., 1988, Rays and caustics as physical objects, in E. Wolf, ed., Progress in optics, 26: Elsevier Science Publ. Co., Inc., 227-348.

Landrø, M., 2001, Discrimination between pressure and fluid saturation changes from time-lapse seismic data: Geophysics, 66, 836-844.

Landrø, M., O. A. Solheim, E. Hilde, B. O. Ekren, and L. K. Strønen, 1999, 
The Gullfaks 4D seismic study: Petroleum Geoscience, 5, 213-226.

Legget, M., N. R. Goulty, and J. E. Kragh, 1993, Study of traveltime and amplitude time-lapse tomography using physical model data: Geophysical Prospecting, 415, 599-619.

Marquering, H., F. A. Dahlen, and G. Nolet, 1999, Three-dimensional sensitivity kernels for finite-frequency traveltimes: The banana-doughnut paradox: Geophysical Journal International, 137, 805-815.

Menke, W., 1989, Geophysical data analysis: Discrete inverse theory: Academic press Inc.

Paulsson, B., J. Meredith, Z. Wang, and J. Fairborn, 1994, The steepbank crosswell seismic project: Reservoir definition and evaluation of steamflood technology in Alberta tar sands: The Leading Edge, 13, 737-747.

Pratt, G. R., 1999, Seismic waveform inversion in the frequency domain, Part I: Theory and verification in a physical scale model: Geophysics, 64, 888 901.

Robertson, J. O. A., Blanch, J. O., and Symes, W. W., 1994, Viscoelastic finite-difference modeling: Geophysics, 59, 1444-1456.

Røste, T., A. Stovas, and M. Landrø, 2006, Estimation of layer thickness and velocity changes using 4D prestack seismic data: Geophysics, 71, no. 6 , S219-S234

Saito, H., D. Nobuoka, H. Azuma, and D. Tanase, 2006, Time-lapse crosswell seismic tomography for monitoring injected $\mathrm{CO}_{2}$ in an onshore aquifer, Nagaoka, Japan: Exploration Geophysics, 37, 132-137.

Snieder, R., 2001, A guided tour of mathematical methods for the physical sciences: Cambridge University Press.

Snieder, R., and C. Chapman, 1998, The reciprocity properties of geometrical spreading: Geophysical Journal International, 132, 82-95.

Snieder, R., and A. Lomax, 1996, Wavefield smoothing and the effect of rough velocity perturbations on arrival times and amplitudes: Geophysical Journal International, 125, 796-812.

Sønneland, L., H. H. Veire, R. Benoit, C. Signer, L. Pedersen, S. Ryan, and S
Colin, 1997, Seismic reservoir monitoring on Gullfaks: The Leading Edge, 16, 1247-1253.

Spetzler, J., 2006, Time-lapse seismic crosswell monitoring of steam injection in tar sand: 76th Annual International Meeting, SEG, Expanded Abstracts, 3120-3124.

Spetzler, J., and R. Snieder, 2001, The effects of small-scale heterogeneity on the arrival time of waves: Geophysical Journal International, 145, 786796.

, 2004, Tutorial: The Fresnel volume and transmitted waves: Geophysics, 69, 653-663.

Spetzler, J., and J. Trampert, 2003, Implementing spectral leakage corrections in global surface wave tomography: Geophysical Journal International, 155, 532-538.

Spetzler, J., Z. Xue, H. Saito, D. Nobuoka, H. Azuma, and O. Nishizawa, 2006, Time-lapse seismic crosswell monitoring of $\mathrm{CO}_{2}$ injected in an onshore sandstone aquifer: 8th Meeting, Society of Exploration Geophysicists of Japan, Proceedings, 336-341.

Tarantola, A., 1987, Inverse problem theory: Elsevier Science Publ. Co., Inc. Trampert, J., and R. Snieder, 1996, Model estimation biased by truncated expansions: Possible artifacts in seismic tomography: Science, 271, 12571260

van Vossen, R., J. O. A. Robertsson, and C. Chapman, 2002, Finite-difference modeling of wave propagation in a fluid-solid configuration: Geophysics, 67, 618-624.

Vesnaver, A. L., F. Accaino, G. Bohm, G. Madrussani, J. Pajchel, G. Rossi, and G. D. Moro, 2003, Case history, time-lapse tomography: Geophysics, $\mathbf{6 8}, 815-823$.

Vesnaver, A., and G. Bohm, 2000, Staggered or adapted grids for seismic tomography?: The Leading Edge, 19,944.

Woodward, M. J., 1992, Wave-equation tomography: Geophysics, 57, $15-26$. 